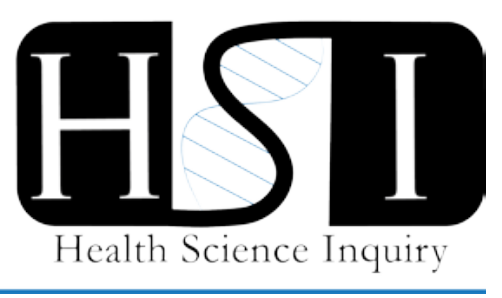

\title{
Enhancing the strategies for diagnosis and treatment of hypertrophic cardiomyopathies
}

\author{
Farigol Hakem Zadeh ${ }^{1,2}$ \\ ${ }^{1}$ Department of Physiology, University of Toronto; ${ }^{2}$ Ted Rogers Centre for Heart Research
}

\begin{abstract}
Hypertrophic cardiomyopathy (HCM) was first diagnosed in humans by Robert Teare in 1958 [1]. HCM is the most prevalent chronic genetic heart disease, affecting every 1 in 500 individuals [2,3]. This disease is defined as the pathological myocyte disarrangement that can lead to thickening of the cardiac walls, which cannot be explained by other cardiac or systemic causes [25]. Some HCM symptoms include chest pain, shortness of breath, irregular heartbeat, blood flow obstruction, inefficient heart pumping, and heart failure $[4,6,7]$. The most severe symptom of HCM is sudden cardiac death (SCD) $[2,5]$. Over the past three decades, several gene mutations that cause HCM have been identified. However, due to the complexity of HCM genetics and symptoms, the diagnosis and management of this disease are challenging. In this article, we will review the discovered human HCM-mutations that are screened during diagnosis, and we will subsequently mention both the advantages and shortcomings of the current HCM management guidelines. Lastly, we will suggest new strategies to enhance the diagnosis and treatment of HCM.
\end{abstract}

Long after the discovery of $\mathrm{HCM}$, the first genetic mutation that causes the disease ( $\beta$-Myosin heavy chain-7 (MYH7)) was linked to it by Seidman et al. in 1990. To date, more than 1500 gene mutations have been associated with HCM $[4,5,7,8]$. This disease is generally autosomal dominant with incomplete penetrance, meaning that not all individuals carrying the genes express HCM traits $[2,5,8]$. The majority of HCM-mutations affect the sarcomeric organization [5]. The known gene mutations linked to HCM are described in Table 1.

These genes can be screened in individuals with symptoms of HCM to detect the source of dysfunction. They become more important when discerning the actual HCM disease from HCM phenocopies, which do not carry HCMgenotype, but have HCM-phenotype as the secondary consequence of other conditions. Determining this distinction helps to diagnose the primary source of the disease and to use the correct protocols for managing patients with $\mathrm{HCM}$ versus those with $\mathrm{HCM}$ phenocopy [8]. However, genetic testing is useful for the diagnosis of around only $60 \%$ of all existing HCMs [7]. We speculate that the advancements in whole-genetic and next-generation sequencing will enhance the discovery of additional HCM-causing mutations. Nevertheless, the incomplete penetrance of $\mathrm{HCM}$ causes variability in disease phenotype. In addition, the age of onset can further complicate the diagnosis [5,7]. Therefore, because of the existence of such variabilities, genetic testing, although necessary for HCM patients, is not the only diagnostic approach that should be relied upon, especially in the cases of asymptomatic individuals [7].

Based on the current guidelines, genetic counselling and screening are required for asymptomatic first-degree relatives of HCM patients [7,8]. The follow-up tests include determining family clinical history, a physical examination, an exercise test, an echocardiogram, and magnetic resonance imaging [5-7]. If the asymptomatic individual is genotype positive for the known HCMmutation, regular follow-ups to monitor disease progression are recommended $[7,8]$. Table 2 provides a summary of clinical approaches undertaken in relation to HCM genotype-phenotype variability.

Current HCM clinical trials show that several biomarkers can be screened in order to identify genotype positive individuals [10]. Therefore, to enhance HCM diagnosis, we suggest that in addition to the current methods, screening for these biomarkers should be added to the guidelines.

Once HCM is diagnosed, a change in lifestyle is required, such as a reduction of athletic activities, in order to decrease the rate of disease progression and the possibility of SCD [2]. Moreover, surgical approaches that remove or obliterate portions of ventricles are used to manage severe cases $[2,3,5]$. In patients with high risk of SCD, the possibilities of implanting a cardioverter-defibrillator or heart transplantation are evaluated $[2,6]$. Furthermore, specific medications, such as $\beta$-blockers, $\mathrm{Ca} 2^{+}$-channel blockers, and anti-arrhythmic drugs, are prescribed [2, 57]. Current studies are looking at drugs that may prevent $\mathrm{HCM}$ in genotype-positive phenotype-negative individuals [8]. Nonetheless, most of the current HCM drugs affect the disease upstream of the sarcomere in cellular mechanisms. These drugs are less selective for the sarcomere and 
Table 1: Gene mutations resulting in $\operatorname{HCM}(4,5,7-9)$. Gene frequencies lower than $3 \%$ are considered rare.

\begin{tabular}{|c|c|c|c|c|}
\hline Family & Subfamily & Protein & Gene & Frequency in Patients \\
\hline \multirow{17}{*}{ 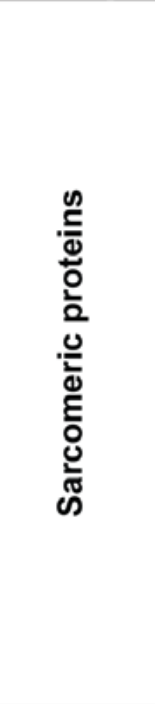 } & \multirow{6}{*}{ 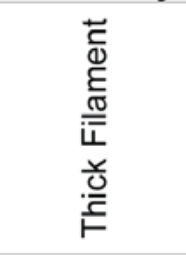 } & $\beta$-Myosin heavy chain & MYH7 & $25-35 \%$ \\
\hline & & Myosin-binding protein C & MYBPC3 & $20-30 \%$ \\
\hline & & Regulatory myosin light chain 2 & MYL2 & $<5 \%$ \\
\hline & & Essential myosin light chain 3 & MYL3 & Rare \\
\hline & & a-Myosin heavy chain & MYH6 & Rare \\
\hline & & Titin & TTN & Rare \\
\hline & \multirow{5}{*}{ 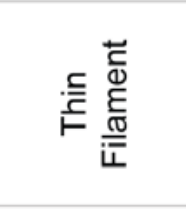 } & Cardiac troponin $\mathrm{T}$ & TNNT2 & $3-5 \%$ \\
\hline & & Cardiac troponin I & TNNI3 & $<5 \%$ \\
\hline & & a-tropomyosin & TPM1 & $<5 \%$ \\
\hline & & Cardiac troponin C & TNNC1 & Rare \\
\hline & & a-cardiac actin & ACTC & Rare \\
\hline & \multirow{6}{*}{$\begin{array}{l}0.00 \\
\text { in } \\
\text { N }\end{array}$} & a-actinin 2 & ACTN2 & Rare \\
\hline & & Myozenin 2 & MYOZ2 & Rare \\
\hline & & Muscle LIM protein & CSRP3 & Rare \\
\hline & & LIM domain binding 3 & LDB3 & Rare \\
\hline & & Telethonin & TCAP & Rare \\
\hline & & Vinculin/metavinculin & VCL & Rare \\
\hline \multirow{4}{*}{ 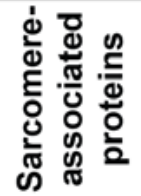 } & \multirow{4}{*}{ 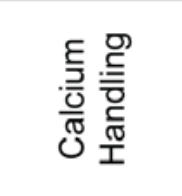 } & Calsequestrin & CASQ2 & Rare \\
\hline & & Junctophilin 2 & JPH2 & Rare \\
\hline & & Phospholamban & PLN & Rare \\
\hline & & Myosin light-chain kinase 2 & MYLK2 & Rare \\
\hline
\end{tabular}

can impact other cellular processes. We think that drug molecules that specifically target proteins within the sarcomere have better therapeutic potential than those that are commonly prescribed and are not specific to HCM.

Table 2: Summary of clinical approaches to HCM based on genotype-phenotype relations.

\begin{tabular}{|c|c|c|c|}
\hline \multirow{2}{*}{\multicolumn{2}{|c|}{ HCM }} & \multicolumn{2}{|c|}{ Phenotype } \\
\hline & & + & - \\
\hline \multirow{2}{*}{ 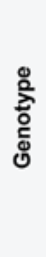 } & + & Medication and therapy & $\begin{array}{c}\text { Annual follow-ups and } \\
\text { Screening }\end{array}$ \\
\hline & ' & $\begin{array}{l}\text { 1) Checking for } \mathrm{HCM} \text { phenocopies } \\
\text { If none found: } \\
\text { 2) Investigating the possibility of } \\
\text { new HCM genetic mutations }\end{array}$ & No follow-up required \\
\hline
\end{tabular}

Based on the current knowledge of HCM, limitations to HCM diagnosis exist, such as the inabilities of diagnosing asymptomatic individuals and all HCM genetic mutations. We suggested that advancements in genetic sequencing and HCM biomarker screening can solve such problems [8]. Furthermore, we noted that the drugs currently available for HCM patients are not HCM-specific. We believe that while new gene-editing approaches and therapeutics are facilitating ways of preventing, reversing or attenuating HCM phenotypes, we should also consider new tools such as biomarkers and HCM-specific drugs in the diagnosis and treatment of this disease.

\section{References}

[1] Liew, A.C., Vassiliou, V.S., Cooper, R., Raphael, C.E. (2017) Hypertrophic Cardiomyopathy-Past, Present and Future. J Clin Med., 6 (12), 475489.

[2] Ellims, A.H. (2017) Hypertrophic cardiomyopathy in the adolescent. Aust Fam Physician, 46 (8), 553557.

[3] Mestres, C.A., Bartel, T., Sorgente, A., Muller, S., Gruner, C., Dearani, J., et al., (2018) Hypertrophic obstructive cardiomyopathy: what, when, why, for whom? Eur J Cardiothorac Surg, 53 (4), 700-707.

[4] Marian, A.J., Braunwald, E. et al., (2018) Hypertrophic Cardiomyopathy: Genetics, Pathogenesis, Clinical Manifestations, Diagnosis, and Therapy. Circ Res, 121 (7), 749-770.

[5] Sen-Chowdhry, S., Tome Esteban, M.T., McKenna, W.J. (2012) Insights and challenges in hypertrophic cardiomyopathy. Herzschrittmacherther Elektrophysiol, 23 (3), 174-185.

[6] Brieler, J., Breeden, M.A., Tucker, J. (2017) Cardiomyopathy: An Overview. Am Fam Physician, 96 (10), 640-646.

[7] Cao, Y., Zhang, P.Y. (2017) Review of recent advances in the management of hypertrophic cardiomyopathy. Eur Rev Med Pharmacol Sci 
[8] Maron, B.J., Maron, M.S., Semsarian, C. (2012) Genetics of hypertrophic cardiomyopathy after 20 years: clinical perspectives. J Am Coll Cardiol, 60 (8), 705715 .

[9] Ren, X., Hensley, N., Brady, M.B., Gao, W.D. (2018) The Genetic and Molecular Bases for Hypertrophic
Cardiomyopathy: The Role for Calcium Sensitization. J Cardiothorac Vasc Anesth, 32 (1), 478-487.

[10] Ho, C.Y., Lopez, B., Coelho-Filho, O.R., Lakdawala, N.K., Cirino, A.L., Jarolim, P., et al. (2010) Myocardial fibrosis as an early manifestation of hypertrophic cardiomyopathy. N Engl J Med, 363 (6), 552-563.

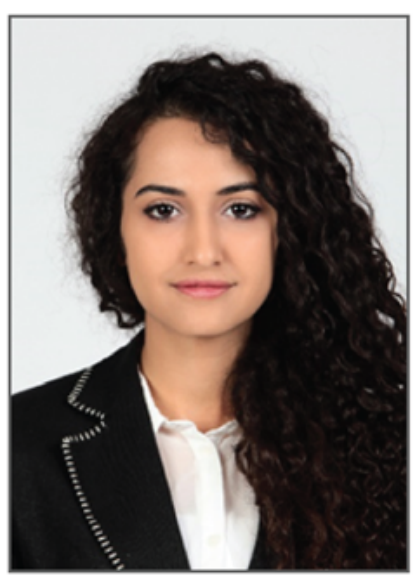

Farigol received her Honours BSc (Physiology) with High Distinction from the University of Toronto. With an extensive family history of cardiovascular diseases and diabetes, she was inspired to contribute to these areas of research. She began her research on myogenic responses in the microvasculature of stroke animal models. She then worked on generating organoid (intestinal buds) from ileal stem cells to study Glucagon-Like peptide- 1 and its effects on insulin secretion. She was the recipient of several academic scholarships including the Queen Elizabeth II Graduate Scholarship. Working at the Ted Rogers Center for Heart Research, Farigol's MSc at the University of Toronto research focused on the regulation of Ca2+-cycling proteins in the heart to target their pathophysiology during cardiomyopathies. Farigol is also passionate about the translational aspect of new interventions in science to clinical medicine. 